\section{OPEN ACCESS}

Edited by:

Laura Sabatino,

Italian National Research Council, Italy

Reviewed by:

Dongfeng Zhang, Qingdao University, China

Cristina Vassalle,

Gabriele Monasterio Tuscany

Foundation (CNR), Italy

*Correspondence:

Mingbing Xiao

xiaomingbing@ntu.edu.cn

Qiang Ma

qiangma@ntu.edu.cn

Specialty section:

This article was submitted to Translational Endocrinology,

a section of the journal

Frontiers in Endocrinology

Received: 14 March 2021

Accepted: 19 July 2021

Published: 12 August 2021

\title{
Association Between C-Peptide Level and Subclinical Myocardial Injury
}

\author{
Ziwei Chen ${ }^{1}$, Jing $\mathrm{He}^{2}$, Qiang $\mathrm{Ma}^{3 *}$ and Mingbing Xiao ${ }^{4 *}$ \\ 1 Department of Cardiology, Affiliated Hospital of Nantong University, Nantong, China, 2 Department of Oncology, Affiliated \\ Hospital of Nantong University, Nantong, China, ${ }^{3}$ School of Public Health, Nantong University, Nantong, China, ${ }^{4}$ Research \\ Center of Clinical Research Center of Clinical Medicine, Affiliated Hospital of Nantong University, Nantong, China
}

Background: Previous studies have confirmed an association between C-peptide levels with the risk of cardiometabolic diseases. However, whether circulating C-peptide was related to subclinical myocardial injury (SC-MI) remains unknown.

Methods: A total of 3,752 participants without a history of cardiovascular diseases were included in our study from National Health and Nutrition Examination Survey III (NHANES III). Multivariable linear regression was performed to explore the correlation between Cpeptide and cardiac injury score (CIIS). Multivariate logistic regression was used to examine the association between C-peptide quartile and SC-MI.

Results: Circulating C-peptide was significantly associated with CIIS ( $\beta: 0.09$, 95\% confidence interval $[\mathrm{Cl}]: 0.00-0.17 ; p=0.041)$. Compared with the lowest quartile, the highest quartile of circulating C-peptide increased a 1.48-fold risk of SC-MI (Odds ratio = 1.66, 95\% Cl: $1.18-1.87 ; p=0.001)$.

Conclusions: The level of C-peptide was independently associated with CIIS and SC-MI, which could serve as a new risk factor of SC-MI.

Keywords: C-peptide, subclinical cardiac injury, NHANES III, association, cross sectional study

\section{BACKGROUND}

Subclinical myocardial injury (SC-MI) is an early cardiac injury without clinically evident coronary heart disease or heart failure $(1,2)$. SC-MI is defined by an electrocardiographic-based scoring system, namely, cardiac infarction/injury score (CIIS) >10 (3). SC-MI was reported to be associated with the progression of coronary heart disease (1) and cardiovascular and all-cause mortality (4).

Abbreviations: SC-MI, subclinical myocardial injury; CIIS, cardiac injury score; NHANES III, National Health and Nutrition Examination Survey III; WBC, white blood cell; RBC, red blood cell; TG, triglyceride; TC, total cholesterol; LDL-C, low-density lipoprotein cholesterol; HDL-C, high-density lipoprotein cholesterol; CRP, C-reactive protein; ALT, alanine aminotransferase; AST, aspartate transaminase glucose; BMI, body mass index. 
Previous studies have reported that physical activity (5), obesity (6), diastolic blood pressure (7), Vitamin D (8) and TyG index

(9) were associated with SC-MI.

C-peptide is a small peptide with 31 amino acids and is released upon insulin secretion to ensure the correct folding of proinsulin $(10,11)$. It is known that $\mathrm{C}$-peptide has been widely used as a biomarker of diabetes diagnosis in clinical practice. Observational studies have found the association between Cpeptide and cardiovascular diseases $(12,13)$. High levels of Cpeptide could increase the risks of atherosclerosis and myocardial infarction $(14,15)$. C-peptide was reported to increase the level of triglyceride and to decrease HDL-C (16). However, C-peptide also inhibited oxidative stress and endothelial apoptosis (17), showing a cardioprotective role. Therefore, it remains unknown that circulating C-peptide level was associated with SC-MI.

In our study, we examined the association between levels of serum C-peptide and SC-MI based on a cross-sectional study.

\section{METHODS}

\section{Study Population}

All participants were included from the US National Health and Nutrition Examination Survey (NHANES III). The NHANES is nationwide multistage survey designed to assess the health and nutritional status of adults and children in the United States (https://www.cdc.gov/nchs/nhanes/index.htm) by the Centers for Disease Control and Prevention (CDC). After excluding individuals with missing circulating C-peptide data, we included 3,752 participants without a history of cardiovascular diseases (Figure 1). The survey protocol was approved by the Institutional Review Board of the CDC.

\section{Covariate Assessments}

The list of covariates includes baseline demographics, the risk factor of cardiovascular diseases, or factors influencing C-peptide levels. Sociodemographic variables, including age, gender, and

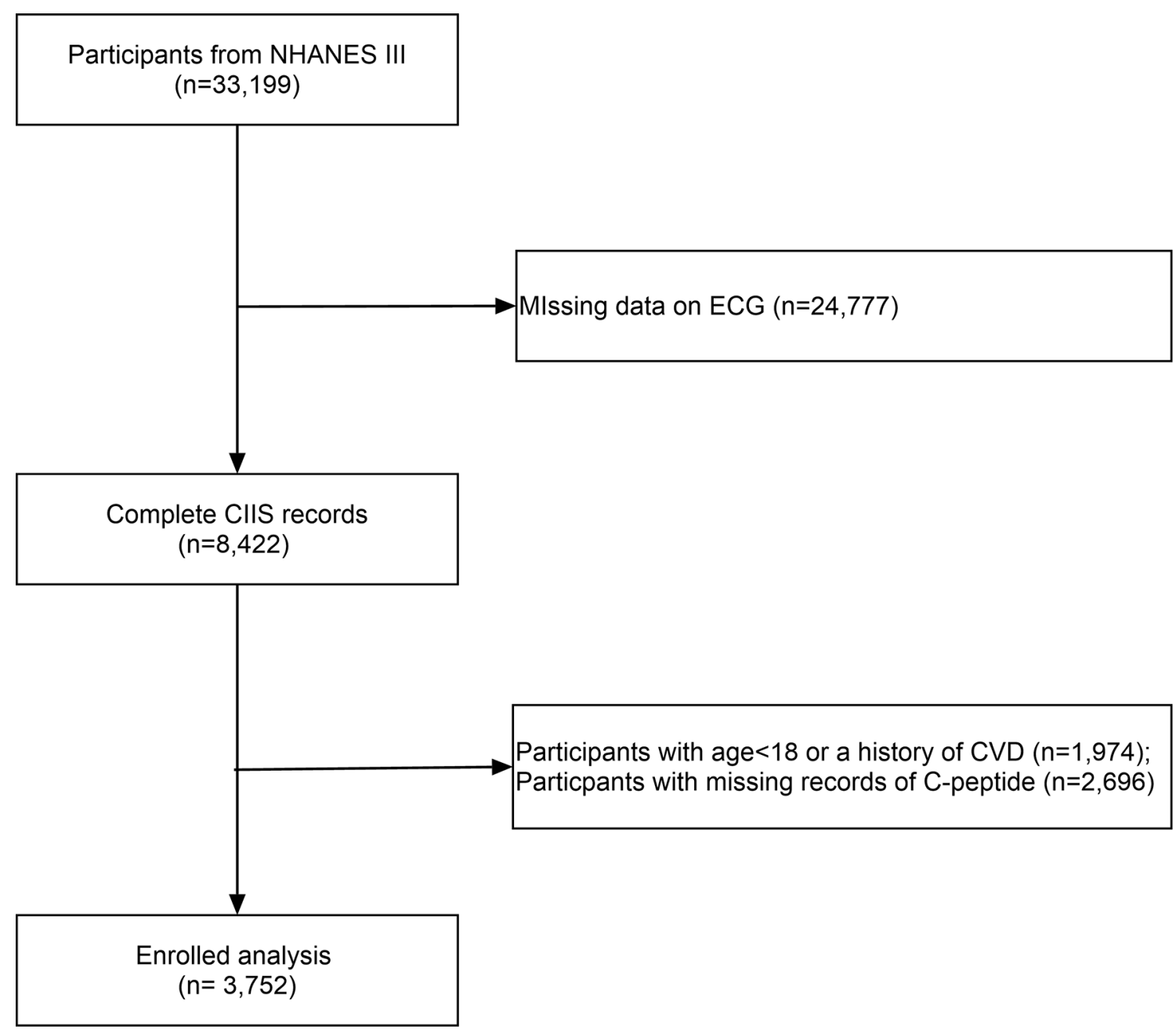

FIGURE 1 | The flow chart of the selection process. 
race, were collected by using standardized questionnaires. The systolic blood pressure, diastolic blood pressure, pulse rate, height, and weight of each participant were obtained from the physical examinations. White blood cell (WBC), red blood cell (RBC), and hemoglobin were obtained by whole blood cell count test. Triglyceride (TG), total cholesterol (TC), low-density lipoprotein cholesterol (LDL-C), high-density lipoprotein cholesterol (HDL-C), C-reactive protein (CRP), creatinine, alanine aminotransferase (ALT), aspartate transaminase (AST), glucose, glycated hemoglobin, and insulin were measured by standard biochemistry assays. Body mass index (BMI, $\mathrm{kg} / \mathrm{m}^{2}$ ) was calculated as weight divided by height squared. Race was classified as non-Hispanic white, non-Hispanic black, Mexican American, and other. Smokers were defined as those who selfreported smoking more than 100 cigarettes during their lifetime, and alcohol users were those who had at least 12 drinks in the last 12 months (18). Physical activity was defined as taking vigorous or moderate activities. Multiple imputation was performed for covariates with missing values.

\section{Circulating C-Peptide Measurement}

Serum C-peptide was measured using a radioimmunoassay (RIA), where the 125I-labeled C-peptide completes with Cpeptide in the specimen for antibody site. Bound and free Cpeptide is separated by adding a second PEG-accelerated double antibody. The antibody-bound fraction is precipitated and counted. The radioactivity is inversely proportional to the quantity of C-peptide in the specimen. Circulating C-peptide was treated as a continuous and a quartile variable to examine its association with CIIS and SC-MI, respectively. The quartile of Cpeptide levels was categorized into four groups: Q1 $(<0.452$ $\mathrm{nmol} / \mathrm{L})$, Q2 (0452-0.737 nmol/L), Q3 (0.737-1.082 nmol/L), and Q4 (>1.082 $\mathrm{nmol} / \mathrm{L})$.

\section{Outcome Definition}

Resting 12-lead electrocardiograms were recorded by experienced technicians with a Marquette MAC 12 system. Analysis of electrocardiograms was achieved through a computerized automated process and visual inspection by a trained technician located in a centralized core laboratory. Briefly, the SC-MI defined from the CIIS rests on a weighted scoring system taking several objective electrocardiographic waveform components related to myocardial injury and ischemia, both discrete and continuous, and generating a riskstratified scoring system $(6,7)$. A combination of 11 discrete and 4 continuous variables are counted to define the final score to evaluate the disease severity levels: CIIS $>20$ for probable injury; CIIS $>15$ for possible injury; CIIS $>10$ for borderline abnormality. SC-MI was defined as a total of CIIS $>10$ (3).

\section{Statistical Analysis}

Sample weights and stratification were incorporated in all analyses because of the complex sampling design of the NHANES data. Categorical variables were expressed as frequencies and percentages. Continuous variables were reported as mean \pm standard deviation or median (interquartile range) if skewed distribution. The difference between groups were compared using one-way
ANOVA for continuous variables or Kruskal-Wallis test if not normalized distributed and chi-square test for categorical variables. The multivariable linear regression was used to explore the association between C-peptide and CIIS (log2-transformed for normality) while the multivariable logistic regression was used to explore the association between C-peptide and SC-MI. To rule out the cofounding factors, we adjusted for age and gender in Model 1. In model 2, we adjusted for age, gender, race, smoking, drinking, smoking, physical activity, and BMI. In model 3, we adjusted for age, gender, race, smoking, drinking, smoking, BMI, TC, TG, CRP, creatinine, AST, ALT, glucose, glycated hemoglobin, and insulin. The variables that were adjusted for were based on the $p$ value $<0.05$ in univariate analysis or the risk factors of cardiometabolic diseases. The restricted cubic spline models with knots at 10th, $50^{\text {th }}$, and 90th percentage were used for the dose-response analysis. Data were analyzed using IBM SPSS 25.0 and R software 3.6. A two-tailed $p$ value $<0.05$ was considered as statistically significant.

\section{RESULTS}

\section{Baseline Characteristics}

A total of 3,752 participants were enrolled in our study with an average age of $60.2 \pm 13.1$, of which 1,778 (47.3\%) were males. Table 1 showed the baseline characteristics grouped by quartiles of circulating C-peptide levels. Participants with high C-peptide levels tend to be old, and male, as well as having higher levels of BMI, TC, TG, LDL-C, CRP, glucose, and glycated hemoglobin.

\section{Association Between C-Peptide Levels and CIIS}

The CIIS was higher with the increased quartile of C-peptide. Multivariable linear regression analysis was used to explore the association between C-peptide and log2-transformed CIIS (Table 2). After adjusting for age and sex, circulating $C$-peptide was positively related to CIIS ( $\beta=0.13,95 \%$ CI: $0.06-0.19 ; p<$ $0.001)$. The linear relationship still existed after adjusting for lifestyles in Model 2 ( $\beta=0.09$, 95\% CI: $0.00-0.17 ; p=0.039)$ and laboratory examinations in Model 3 ( $\beta=0.09$, 95\% CI: $0.00-0.17$; $p$ $=0.041$ ). Compared with the lowest quartile, the highest quartile of C-peptide was positively associated with CIIS across three models.

\section{Association Between C-Peptide Levels and SC-MI}

The prevalence of SC-MI was 31.6\%, 31.3\%, 38.6\%, and $43.6 \%$ across quartiles, respectively. Table 3 summarizes the results of multivariable logistic regression between C-peptide quartiles and SC-MI. Compared to the lowest quartile, the highest quartile was significantly associated with SC-MI in model 1 adjusted for sociodemographics $(\mathrm{OR}=1.59,95 \% \mathrm{CI}: 1.31-1.92 ; p<0.001)$, and this association remained statistically significant in Model 2 $(\mathrm{OR}=1.48,95 \% \mathrm{CI}: 1.18-1.86 ; p=0.001)$ and Model $3(\mathrm{OR}=$ $1.48,95 \% \mathrm{CI}: 1.18-1.87 ; p=0.001)$. In addition, one-unit increase of C-peptide was associated with 1.27-fold higher risk of SC-MI (OR $=1.27,95 \%$ CI:1.08-1.50; $p=0.004)$. 
TABLE 1 | Baseline characteristics of study participants across serum C-peptide categories.

\begin{tabular}{|c|c|c|c|c|c|}
\hline & Q1 $(\leq 0.452)$ & Q2 (0.452-0.737) & Q3 (0.737-1.082) & Q4 ( $\geq 1.082)$ & $p$-value \\
\hline$n$ & 941 & 938 & 936 & 937 & \\
\hline Age (years) & $58.4 \pm 13.3$ & $59.6 \pm 12.9$ & $61.3 \pm 13.4$ & $61.4 \pm 12.8$ & $<0.001$ \\
\hline Male (\%) & 377 (40.1) & $447(47.7)$ & $472(50.4)$ & $480(51.2)$ & $<0.001$ \\
\hline Race (\%) & & & & & $<0.001$ \\
\hline Non-Hispanic white & $521(55.4)$ & $527(56.2)$ & $484(51.7)$ & $476(50.8)$ & \\
\hline Non-Hispanic black & $249(26.5)$ & $198(21.1)$ & $200(21.4)$ & $172(18.4)$ & \\
\hline Mexican-American & $142(15.1)$ & $179(19.1)$ & $220(23.5)$ & 252 (26.9) & \\
\hline Other & $29(3.1)$ & $34(3.6)$ & $32(3.4)$ & 37 (3.9) & \\
\hline Smoking (\%) & $312(33.2)$ & 303 (32.3) & 265 (28.3) & $202(21.6)$ & $<0.001$ \\
\hline Drinking (\%) & $511(54.3)$ & $523(55.8)$ & $549(58.7)$ & $523(55.8)$ & 0.467 \\
\hline Physical activity (\%) & $580(61.7)$ & $513(54.7)$ & $478(51.1)$ & $461(49.2)$ & $<0.001$ \\
\hline BMI $\left(\mathrm{kg} / \mathrm{m}^{2}\right)$ & $24.0 \pm 4.2$ & $26.5 \pm 4.2$ & $28.5 \pm 5.2$ & $31.2 \pm 6.1$ & $<0.001$ \\
\hline $\mathrm{sBP}(\mathrm{mmHg})$ & $127.6 \pm 19.8$ & $130.9 \pm 18.6$ & $134.9 \pm 19.1$ & $136.2 \pm 18.3$ & $<0.001$ \\
\hline $\mathrm{dBP}(\mathrm{mmHg})$ & $58.3 \pm 6.8$ & $58.1 \pm 7.1$ & $58.1 \pm 7.2$ & $57.9 \pm 7.4$ & 0.680 \\
\hline Pulse (bpm) & $73.3 \pm 11.4$ & $74.7 \pm 11.9$ & $76.0 \pm 12.3$ & $79.0 \pm 13.1$ & $<0.001$ \\
\hline WBC, $10^{9} / \mathrm{L}$ & $6.6 \pm 2.0$ & $6.9 \pm 2.2$ & $7.3 \pm 3.1$ & $7.8 \pm 2.6$ & $<0.001$ \\
\hline RBC, $10^{12} / \mathrm{L}$ & $4.5 \pm 0.4$ & $4.6 \pm 0.4$ & $4.7 \pm 0.4$ & $4.8 \pm 0.5$ & $<0.001$ \\
\hline Hemoglobin (g/dl) & $13.5 \pm 1.4$ & $14.0 \pm 1.4$ & $14.1 \pm 1.4$ & $14.4 \pm 1.5$ & $<0.001$ \\
\hline Total cholesterol (mg/dl) & 206 (53) & $216(51)$ & $216(52)$ & $214(52)$ & $<0.001$ \\
\hline Triglycerides (mg/dl) & $86(49)$ & $113(61)$ & $139(86)$ & $166(91)$ & $<0.001$ \\
\hline LDL-C (mg/dl) & $127(49)$ & $140(44)$ & $138(47)$ & $136(47)$ & $<0.001$ \\
\hline $\mathrm{HDL}-\mathrm{C}(\mathrm{mg} / \mathrm{dl})$ & $57(23)$ & $51(20)$ & $46(16)$ & $42(14)$ & $<0.001$ \\
\hline C-reactive protein (mg/dl) & $0.21(0.1)$ & $0.21(0.2)$ & $0.21(0.3)$ & $0.33(0.5)$ & $<0.001$ \\
\hline Creatinine (mg/dl) & $1.05 \pm 0.18$ & $1.08 \pm 0.22$ & $1.11 \pm 0.27$ & $1.18 \pm 0.55$ & $<0.001$ \\
\hline AST (U/L) & $21.7 \pm 13.8$ & $21.2 \pm 11.6$ & $22.3 \pm 16.9$ & $24.6 \pm 18.7$ & $<0.001$ \\
\hline $\mathrm{ALT}(\mathrm{U} / \mathrm{L})$ & $13.6 \pm 9.9$ & $15.1 \pm 9.5$ & $17.6 \pm 14.2$ & $21.7 \pm 17.8$ & $<0.001$ \\
\hline Glucose (mg/dl) & $90(13)$ & $96(14)$ & $101(17)$ & $108(24)$ & $<0.001$ \\
\hline Glycated hemoglobin (\%) & $5.5 \pm 1.0$ & $5.7 \pm 1.1$ & $5.9 \pm 1.3$ & $6.2 \pm 1.5$ & $<0.001$ \\
\hline Insulin $(\mu \mathrm{U} / \mathrm{ml})$ & $5.6(2.5)$ & $7.9(2.9)$ & $11(4.4)$ & $17.8(9.3)$ & $<0.001$ \\
\hline CIIS & $6.2(8.7)$ & $6.6(7.8)$ & $7.2(9.6)$ & $8.5(11.2)$ & $<0.001$ \\
\hline SC-MI & 297 (31.6) & $646(31.1)$ & $361(38.6)$ & $528(43.6)$ & $<0.001$ \\
\hline
\end{tabular}

TABLE 2 | Multivariable linear regression between C-peptide and log2-CIIS.

\begin{tabular}{|c|c|c|c|c|c|c|}
\hline & \multicolumn{2}{|c|}{ Model 1} & \multicolumn{2}{|c|}{ Model 2} & \multicolumn{2}{|c|}{ Model 3} \\
\hline & $\beta(95 \% \mathrm{Cl})$ & $p$ & $\beta(95 \% \mathrm{Cl})$ & $p$ & $\beta(95 \% \mathrm{Cl})$ & $p$ \\
\hline Q1 & Ref & & Ref & & Ref & \\
\hline Q2 & $0.01[-0.10,0.11]$ & 0.892 & $-0.01[-0.11,0.10]$ & 0.863 & $-0.01[-0.11,0.10]$ & 0.856 \\
\hline Q3 & $0.13[0.02,0.23]$ & 0.015 & $0.10[-0.01,0.21]$ & 0.074 & $0.10[-0.01,0.21]$ & 0.083 \\
\hline Q4 & $0.21[0.10,0.31]$ & $<0.001$ & $0.16[0.04,0.28]$ & 0.011 & $0.16[0.03,0.28]$ & 0.015 \\
\hline Per one-unit & $0.13[0.06,0.19]$ & $<0.001$ & $0.09[0.00,0.17]$ & 0.039 & $0.09[0.00,0.17]$ & 0.041 \\
\hline
\end{tabular}

OR, odds ratio; $\mathrm{Cl}$, confidence interval; BMI, body mass index; TC, total cholesterol; TG, triglyceride, CRP, c-reactive protein.

Model 1 was adjusted for age and gender.

Model 2 was adjusted for age, gender, race, smoking, drinking, physical activity, BMI, TC, TG, CRP, and creatinine.

Model 3 was adjusted for age, gender, race, smoking, drinking, physical activity, BMI, TC, TG, CRP, creatinine, glucose, glycated hemoglobin, and insulin.

To explore the nonlinear relationship, we performed doseresponse analysis based on restricted cubic spline models (Figure 2). It suggested that $\mathrm{C}$-peptide was linearly and positively related to SC-MI $(p$ for nonlinearity $=0.860$ ).

\section{Subgroup Analysis}

Subgroup analysis was performed to explore the potential factors modifying the association between C-peptide and SC-MI. The association of C-peptide with SC-MI was consistent across age and gender (Table 4). Besides, we found that a higher level of Cpeptide increased the risk of SC-MI across the disease severity (Table 5). However, it was only significant in the borderline abnormality group $(p=0.031)$.

\section{DISCUSSION}

Our study confirmed that circulating C-peptide level was independently associated with electrocardiographic subclinical myocardial injury after adjusting for cardiovascular risk factors and glucose metabolism-related biomarkers.

Previous studies have shown the association between Cpeptide and cardiovascular disease (19). Michelle et al. examined the effect of C-peptide on atherosclerosis and found that high C-peptide levels were related with increased lipid deposits and smooth muscle cell proliferation in the vessel wall, contributing to atherosclerosis (12). Antonio Cabrera de Leon et al. found that elevated C-peptide was 
TABLE 3 | Multivariable logistic regression between C-peptide categories and subclinical myocardial injury.

\begin{tabular}{|c|c|c|c|c|c|c|}
\hline & \multicolumn{2}{|c|}{ Model 1} & \multicolumn{2}{|c|}{ Model 2} & \multicolumn{2}{|c|}{ Model 3} \\
\hline Q1 & Ref & & Ref & & Ref & \\
\hline Q2 & $0.95[0.78,1.16]$ & 0.619 & $0.92[0.75,1.13]$ & 0.426 & $0.92[0.75,1.12$ & 0.414 \\
\hline Q3 & $1.29[1.06,1.56]$ & 0.010 & $1.23[1.00,1.51]$ & 0.053 & $1.22[0.99,1.51]$ & 0.056 \\
\hline Q4 & $1.59[1.31,1.92]$ & $<0.001$ & $1.48[1.18,1.86]$ & 0.001 & $1.48[1.18,1.87]$ & 0.001 \\
\hline Per one-unit & $1.35[1.18,1.54]$ & $<0.001$ & $1.25[1.07,1.47]$ & 0.005 & $1.27[1.08,1.50]$ & 0.004 \\
\hline
\end{tabular}

Model 1 was adjusted for age and gender.

Model 2 was adjusted for age, gender, race, smoking, drinking, physical activity, BMI, TC, TG, CRP, and creatinine.

Model 3 was adjusted for age, gender, race, smoking, drinking, physical activity, BMI, TC, TG, CRP, creatinine, glucose, glycated hemoglobin, and insulin.

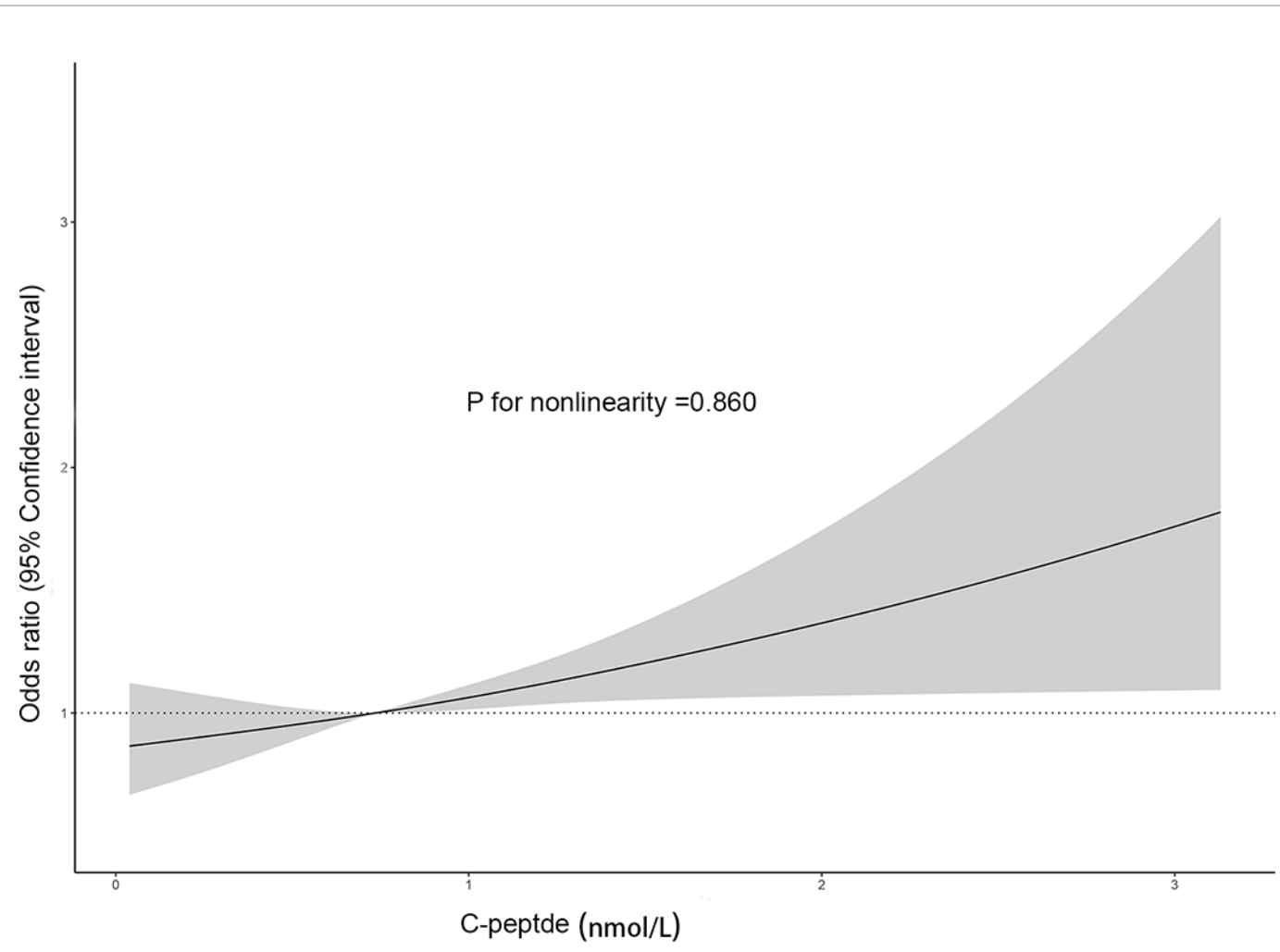

FIGURE 2 | The dose-response relation between C-peptide and SC-MI.

TABLE 4 | Subgroup analysis between C-peptide and SC-MI.

\begin{tabular}{|c|c|c|c|}
\hline & OR (95\% Cl) & $p$ & $p$ for interaction \\
\hline Male & $1.26[1.00,1.62]$ & 0.062 & \\
\hline Female & $1.21[0.95,1.53]$ & 0.121 & \\
\hline Age & & & 0.262 \\
\hline$\leq 65$ & $1.43[1.12,1.84]$ & 0.005 & \\
\hline$>65$ & $1.21[0.97,1.57]$ & 0.113 & \\
\hline
\end{tabular}

TABLE 5 | The association between C-peptide and severity of SC-MI.

\begin{tabular}{lccc}
\hline Disease severity & Cases & OR (95\% Cl) & P \\
\hline Borderline abnormality; $10<\mathrm{C} \| \mathrm{S} \leq 15$ & 671 & $1.24[1.02,1.51]$ & 0.031 \\
Possible injury; $15<\mathrm{C} \| \mathrm{S} \leq 20$ & 391 & $1.12[0.90,1.37]$ & 0.293 \\
Probable injury; CIIS>20 & 273 & $1.15[0.89,1.45]$ & 0.259
\end{tabular}


associated with the incidence of myocardial infarction and coronary artery disease in the general population (20). What is more, Min et al. showed an association between serum Cpeptide levels and all-cause and cause-specific mortality among adults without diabetes (21). Furthermore, we have observed a strong association of C-peptide levels with CIIS and SC-MI in our study, which could contribute to early diagnosis and intervention.

The underlying mechanisms for this association were complicated. The C-peptide has been related to increasing triglyceride levels while decreasing HDL-C levels, which were negatively correlated to cardiovascular death (16). However, evidence also indicated that C-peptide has an antiinflammatory role (22) and anti-oxidative role (23). So, it needs more prospective clinical trials to determine whether the increased C-peptide in the SC-MI was causal or compensatory.

Certain limitations need to be taken into consideration in the interpretation of our study. Our study was designed by a crosssectional scheme, only revealing the correlation between $\mathrm{C}$ peptide and SC-MI. Besides, only baseline C-peptide was included in our study, and it may be more meaningful to examine the change of $\mathrm{C}$-peptide.

\section{CONCLUSION}

We observed an association between high C-peptide levels and SC-MI in the general population.

\section{REFERENCES}

1. O'Neal WT, Shah AJ, Efird JT, Rautaharju PM, Soliman EZ. Subclinical Myocardial Injury Identified by Cardiac Infarction/Injury Score and the Risk of Mortality in Men and Women Free of Cardiovascular Disease. Am J Cardiol (2014) 114(7):1018-23. doi: 10.1016/j.amjcard.2014.06.032

2. Rubin J, Matsushita K, Ballantyne CM, Hoogeveen R, Coresh J, Selvin E. Chronic Hyperglycemia and Subclinical Myocardial Injury. J Am Coll Cardiol (2012) 59(5):484-9. doi: 10.1016/j.jacc.2011.10.875

3. Rautaharju PM, Warren JW, Jain U, Wolf HK, Nielsen CL. Cardiac Infarction Injury Score: An Electrocardiographic Coding Scheme for Ischemic Heart Disease. Circulation (1981) 64(2):249-56. doi: 10.1161/01.cir.64.2.249

4. Richardson K, Engel G, Yamazaki T, Chun S, Froelicher VF. Electrocardiographic Damage Scores and Cardiovascular Mortality. Am Heart J (2005) 149(3):458-63. doi: 10.1016/j.ahj.2004.06.025

5. German C, Ahmad MI, Li Y, Soliman EZ. Relations Between Physical Activity, Subclinical Myocardial Injury, and Cardiovascular Mortality in the General Population. Am J Cardiol (2020) 125(2):205-9. doi: 10.1016/ j.amjcard.2019.08.031

6. Vasim I, Ahmad MI, Mongraw-Chaffin M, Soliman EZ. Association of Obesity Phenotypes With Electrocardiographic Subclinical Myocardial Injury in the General Population. Clin Cardiol (2019) 42(3):373-8. doi: $10.1002 /$ clc. 23155

7. Waits GS, O’Neal WT, Sandesara PB, Li Y, Shah AJ, Soliman EZ. Association Between Low Diastolic Blood Pressure and Subclinical Myocardial Injury. Clin Res Cardiol (2018) 107(4):312-8. doi: 10.1007/s00392-017-1184-0

8. Ahmad MI, Chevli PA, Li Y, Soliman EZ. Vitamin D Deficiency and Electrocardiographic Subclinical Myocardial Injury: Results From National

\section{DATA AVAILABILITY STATEMENT}

The datasets presented in this study can be found in online repositories. The names of the repository/repositories and accession number(s) can be found below: NHANES III.

\section{ETHICS STATEMENT}

The studies involving human participants were reviewed and approved by Affiliated Hospital of Nantong University. The patients/participants provided their written informed consent to participate in this study.

\section{AUTHOR CONTRIBUTIONS}

$\mathrm{XMB}$ and $\mathrm{MQ}$ designed this study, ZC wrote the manuscript. JH performed the experiments. All authors contributed to the article and approved the submitted version.

\section{FUNDING}

This study was supported by the Municipal Natural Science Foundation of Nantong (Nos. MS22019004, YYZ-16012 and JCZ20207) and the social science fund of Jiangsu Province (No. 19GLB026).

Health and Nutrition Examination Survey-III. Clin Cardiol (2018) 41 (11):1468-73. doi: 10.1002/clc.23078

9. Liu Y, Wu M, Xu J, Sha D, Xu B, Kang L. Association Between Triglyceride and Glycose (TyG) Index and Subclinical Myocardial Injury. Nutr Metab Cardiovasc Dis (2020) 30(11):2072-6. doi: 10.1016/j.numecd.2020.06.019

10. Shaw JA, Shetty P, Burns KD, Fergusson D, Knoll GA. C-Peptide as a Therapy for Kidney Disease: A Systematic Review and Meta-Analysis. PloS One (2015) 10(5):e0127439. doi: 10.1371/journal.pone.0127439

11. Yosten GL, Maric-Bilkan C, Luppi P, Wahren J. Physiological Effects and Therapeutic Potential of Proinsulin C-Peptide. Am J Physiol Endocrinol Metab (2014) 307(11):E955-68. doi: 10.1152/ajpendo.00130.2014

12. Alves MT, Ortiz MMO, Dos Reis G, Dusse LMS, Carvalho MDG, Fernandes AP, et al. The Dual Effect of C-Peptide on Cellular Activation and Atherosclerosis: Protective or Not? Diabetes Metab Res Rev (2019) 35(1): e3071. doi: 10.1002/dmrr.3071

13. Patel N, Taveira TH, Choudhary G, Whitlatch H, Wu WC. Fasting Serum CPeptide Levels Predict Cardiovascular and Overall Death in Nondiabetic Adults. J Am Heart Assoc (2012) 1(6):e003152. doi: 10.1161/JAHA.112.003152

14. Abdullah A, Hasan H, Raigangar V, Bani-Issa W. C-Peptide Versus Insulin: Relationships With Risk Biomarkers of Cardiovascular Disease in Metabolic Syndrome in Young Arab Females. Int J Endocrinol (2012) 2012:420792. doi: $10.1155 / 2012 / 420792$

15. Harnishsingh B, Rama B. Is C-Peptide a Predictor of Severity of Coronary Artery Disease in Metabolic Syndrome? An Observational Study. Indian Heart J (2018) 70 Suppl 3:S105-9. doi: 10.1016/j.ihj.2018.07.005

16. Li Y, Zhao D, Li Y, Meng L, Enwer G. Serum C-Peptide as a Key Contributor to Lipid-Related Residual Cardiovascular Risk in the Elderly. Arch Gerontol Geriatr (2017) 73:263-8. doi: 10.1016/j.archger.2017.05.018 
17. Bhatt MP, Lim YC, Hwang J, Na S, Kim YM, Ha KS. C-Peptide Prevents Hyperglycemia-Induced Endothelial Apoptosis Through Inhibition of Reactive Oxygen Species-Mediated Transglutaminase 2 Activation. Diabetes (2013) 62(1):243-53. doi: 10.2337/db12-0293

18. Liao S, Zhang J, Shi S, Gong D, Lu X, Cheang I, et al. Association of Aldehyde Exposure With Cardiovascular Disease. Ecotoxicol Environ Saf (2020) 206:111385. doi: 10.1016/j.ecoenv.2020.111385

19. Vasic D, Walcher D. C-Peptide: A New Mediator of Atherosclerosis in Diabetes. Mediators Inflamm (2012) 2012:858692. doi: 10.1155/2012/858692

20. Cabrera de Leon A, Oliva Garcia JG, Marcelino Rodriguez I, Almeida Gonzalez D, Aleman Sanchez JJ, Brito Diaz B, et al. C-Peptide as a Risk Factor of Coronary Artery Disease in the General Population. Diabetes Vasc Dis Res (2015) 12(3):199-207. doi: 10.1177/1479164114564900

21. Li Y, Li Y, Meng L, Zheng L. Association Between Serum C-Peptide as a Risk Factor for Cardiovascular Disease and High-Density Lipoprotein Cholesterol Levels in Nondiabetic Individuals. PloS One (2015) 10(1):e112281. doi: 10.1371/journal.pone.0112281

22. Kao RLC, Xu X, Xenocostas A, Parry N, Mele T, Martin CM, et al. C-Peptide Attenuates Acute Lung Inflammation in a Murine Model of Hemorrhagic Shock and Resuscitation by Reducing Gut Injury. J Trauma Acute Care Surg (2017) 83(2):256-62. doi: 10.1097/TA.0000000000001539
23. Ragy MM, Ahmed SM. Protective Effects of Either C-Peptide or L-Arginine on Pancreatic Beta-Cell Function, Proliferation, and Oxidative Stress in Streptozotocin-Induced Diabetic Rats. J Cell Physiol (2019) 234(7):1150010. doi: $10.1002 /$ jcp. 27808

Conflict of Interest: The authors declare that the research was conducted in the absence of any commercial or financial relationships that could be construed as a potential conflict of interest.

Publisher's Note: All claims expressed in this article are solely those of the authors and do not necessarily represent those of their affiliated organizations, or those of the publisher, the editors and the reviewers. Any product that may be evaluated in this article, or claim that may be made by its manufacturer, is not guaranteed or endorsed by the publisher.

Copyright $\odot 2021$ Chen, He, Ma and Xiao. This is an open-access article distributed under the terms of the Creative Commons Attribution License (CC BY). The use, distribution or reproduction in other forums is permitted, provided the original author(s) and the copyright owner(s) are credited and that the original publication in this journal is cited, in accordance with accepted academic practice. No use, distribution or reproduction is permitted which does not comply with these terms. 